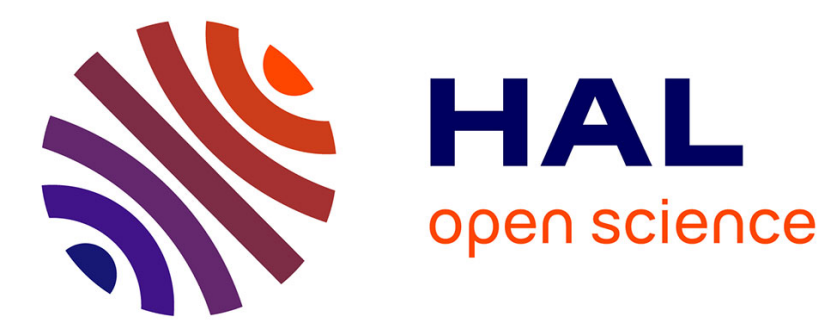

\title{
Les réseaux judiciaires en Iraq à l'époque abbasside
}

Mathieu Tillier

\section{To cite this version:}

Mathieu Tillier. Les réseaux judiciaires en Iraq à l'époque abbasside. D. Coulon, Ch. Picard, D. Valérian. Espaces et réseaux en Méditerranée, vol. II. La formation des réseaux, Bouchène, pp.91107, 2010. hal-00587356

\section{HAL Id: hal-00587356 https://hal.science/hal-00587356}

Submitted on 20 Apr 2011

HAL is a multi-disciplinary open access archive for the deposit and dissemination of scientific research documents, whether they are published or not. The documents may come from teaching and research institutions in France or abroad, or from public or private research centers.
L'archive ouverte pluridisciplinaire HAL, est destinée au dépôt et à la diffusion de documents scientifiques de niveau recherche, publiés ou non, émanant des établissements d'enseignement et de recherche français ou étrangers, des laboratoires publics ou privés. 


\title{
Les réseaux judiciaires en Iraq à l'époque abbasside
}

\author{
par
}

Mathieu Tillier

Parmi les institutions judiciaires qui se développèrent aux premiers siècles de l'Islam, celle du cadi entretint les rapports les plus ambigus avec le pouvoir souverain. Tandis que la justice des gouverneurs locaux ou de leurs șāḥib-s al-šurța (chefs de la police) était avant tout celle de militaires tenus de maintenir l'ordre et relevait d'une pratique gouvernementale qui ne fit pas l'objet d'une théorisation systématique par les fuqahà', celle des cadis apparut rapidement comme la justice islamique par excellence, à la fois enserrée dans une stricte hiérarchie institutionnelle dominée par le califat et régie par une théorie juridique qui lui échappait largement. Cette double relation à l'État et au fiqh rend particulièrement malaisée l'étude de la formation et du fonctionnement de l'institution. Les liens entre la judicature et l'État islamique ne peuvent cependant être mesurés à travers la seule activité locale des tribunaux. Leur fonctionnement en réseau, par la mise en œuvre d'une procédure épistolaire entre cadis (kitāb al-qā expressions les plus significatives de la continuité étatique et de l'unité territoriale du dār al-islām. Nous proposons de nous interroger ici sur la constitution de tels réseaux, sur leur nature et sur leur mode de fonctionnement en Iraq entre le $\mathrm{II}^{\mathrm{e}} / \mathrm{VIII}^{\mathrm{e}}$ et $\mathrm{le} \mathrm{IV}^{\mathrm{e}} / \mathrm{x}^{\mathrm{e}}$ siècle. La réglementation des procédures judiciaires à distance, en particulier, fut-elle imposée par un pouvoir soucieux de contrôler tous les rouages de l'institution judiciaire ? Ou celle-ci surgitelle au contraire d'une réflexion privée ? La communication entre cadis empruntait-elle des réseaux étatiques permanents ou, à l'inverse, fut-elle convoyée par des voies extraétatiques? Il apparaîtra que ces questions peuvent difficilement faire l'objet de réponses tranchées et que la continuité judiciaire reposait sur un subtil partage de compétences entre l'État et le domaine privé.

\section{Maillage judiciaire et compétences territoriales des cadis}

À l'époque abbasside, l'administration judiciaire ne couvrait pas le dār al-islām de manière uniforme. Le califat - dont procédait en principe la désignation des cadis - ne conçut jamais de quadrillage institutionnel du territoire où s'exerçait sa souveraineté. Pour des raisons historiques, liées au regroupement initial des Musulmans dans des implantations limitées, la carte judiciaire se présentait comme une série de districts dispersés. D'une institution locale, supervisée par les gouverneurs, la judicature avait acquis une envergure impériale sous al-Manșūr et ses successeurs; elle n'en conservait pas moins une structure éclatée en une multitude de circonscriptions non contiguës ${ }^{1}$. Les cadis étaient nommés par le souverain, le calife, qui leur déléguait une partie de ses pouvoirs et leur donnait mandat - par oral et/ou par écrit - de rendre la justice en son nom sur un district. Mais dans les modèles d'actes d'investiture ayant survécu pour l'époque abbasside, les frontières des districts ne sont pas spécifiées ${ }^{2}$. Il semble qu'à l'instar de l'image véhiculée par les chroniques ou les dictionnaires biographiques, le pouvoir désignait généralement un cadi sur une ville ou, exceptionnellement, sur une partie de ville (Bagdad fut divisée en trois

\footnotetext{
${ }^{1}$ M. Tillier, Les cadis d'Iraq à l'époque 'abbāside : organisation judiciaire et rapports au pouvoir (132/750-334/945), Thèse de doctorat, Université Lyon 2, 2004, p. 272.

${ }^{2}$ Voir par exemple Qudāma b. Ğa far, Kitāb al-ḩarāğ wa-șināat al-kitāba, éd. M.H. al-Zabīì̄, Bagdad, 1981, p. 39.
} 
circonscriptions judiciaires jusqu'au $\mathrm{IV}^{\mathrm{e}} / \mathrm{x}^{\mathrm{e}}$ siècle $\left.{ }^{3}\right)$. Aucun indice que l'État ait entrepris de décrire les limites précises de ces ressorts ne nous est parvenu.

L'étendue des districts judiciaires fut en réalité définie " d'en bas », à travers la réflexion

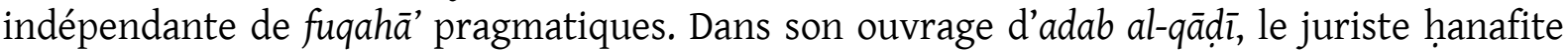
al-Hुașạāf (m. 261/874) se demanda jusqu'à quelle distance un cadi pouvait convoquer un justiciable contre qui plainte avait été déposée. Il était nécessaire, répondit-il, que le défendeur puisse venir à l'audience et retourner chez lui en l'espace d'une journée ; au-delà, des preuves devaient être au préalable produites à son encontre, afin que le cadi soit sûr de ne pas le convoquer en vain ${ }^{4}$. Telle qu'envisagée par les juristes hanafites, la circonscription judiciaire dépassait la ville où siégeait le cadi pour englober les campagnes environnantes, tout en conservant des limites assez floues. À l'intérieur d'un premier cercle, au rayon d'une demi-journée de route (environ 10 milles, ou $20 \mathrm{Km}^{5}$ ), les populations musulmanes relevaient sans ambiguïté du cadi siégeant au centre. Au gré des circonstances, l'autorité du cadi pouvait s'étendre au-delà, mais sans limite précise. Le ressort du cadi se définissait moins en terme de territoire géographiquement délimité qu'en terme de zone d'influence centripète. Les principales villes et leurs territoires limitrophes constituaient des espaces fortement soumis au cadi; plus on s'éloignait du centre, plus son pouvoir décroissait, jusqu'à ce que l'on pénètre dans la zone d'influence d'une autre ville et d'un autre cadi.

$\mathrm{Au}$ début $\mathrm{du} \mathrm{III}^{\mathrm{e}} / \mathrm{IX}^{\mathrm{e}}$ siècle, seules les plus grandes villes d'Iraq, à l'instar de Bașra, Küfa, Wāsiț, al-Anbār et Bagdad - c'est-à-dire les cités peuplées d'une forte majorité de Musulmans et possédant une grande mosquée -, disposaient d'un cadi. La judicature était liée à une plus large présence institutionnelle de l'État islamique, dont une des principales expressions architecturales était la grande mosquée - sa construction était soumise à l'autorisation du calife ${ }^{6}$ - et dont la manifestation symbolique essentielle était la hut ba, lors de la prière du vendredi. Aussi la carte des circonscriptions judiciaires iraqiennes présentait-elle l'image d'une peau de léopard, dont les vides ne se comblèrent que très lentement, au gré de l'islamisation du territoire iraqien - l'établissement de structures étatiques islamiques s'avérant indispensable au maintien de la cohésion sociale lorsque les institutions non-musulmanes ne remplirent plus leurs fonctions auprès de la majorité ${ }^{7}$. Au caractère morcelé de l'espace judiciaire venait s'ajouter la forte mobilité des justiciables, amenés à circuler pour des motifs commerciaux ou académiques. La question de la continuité géographique de la judicature s'imposa donc rapidement: l'autorité d'un cadi s'éteignant de facto au-delà d'une certaine distance, comment la justice pouvait-elle être préservée si un accusé sortait du district où il avait commis son infraction?

Les compétences limitées du cadi, conjuguées aux règles strictes de la procédure ordinaire, entravaient considérablement la poursuite d'un défendeur éloigné. À l'extérieur de sa circonscription, le cadi redevenait un homme du commun et perdait toute capacité de prononcer un jugement ${ }^{8}$. Par ailleurs, la procédure exigeait normalement la présence conjointe du demandeur et du défendeur devant le cadi pour que ce dernier entende la

\footnotetext{
${ }^{3}$ Ș.A. al-'Alī, "Quḍāt Baġdād fì l-'așr al-'abbāsī. Dirāsa fì-l-idāra l-islāmiyya », Mağallat al-Mağma' al-'ilmī al-'irāqī, 18 (1969), p. 192-208.

${ }^{4}$ Al-Hुașșāf, Adab al-qāẹ̄̄, éd. F. Ziyāda, Le Caire, 1978, p. 250. Cf. Ibn al-Qāṣṣ, Adab al-qāọī, éd. A.F. al-Mazīdī, Beyrouth, 2007, p. 110.

${ }^{5}$ Selon A.J. Silverstein (Postal Systems in the Pre-Modern Islamic World, New York, 2007, p. 94), la marhala - ou distance parcourue par une caravane en une journée - représentait $35 \mathrm{Km}$ en moyenne.

${ }^{6}$ I.M. Lapidus, « Muslim Cities and Islamic Societies », dans I.M. Lapidus (éd.), Middle Eastern Cities. A Symposium on Ancient, Islamic, and Contemporary Middle Eastern Urbanism, Berkley-Los Angeles, 1969, p. 71.

${ }^{7}$ Tillier, Les cadis d'Iraq à l'époque 'abbāside, p. 272, 291.

${ }^{8}$ B. Johansen, «Formes de langage et fonctions publiques : stéréotypes, témoins et offices dans la preuve par l'écrit en droit musulman », Arabica, 44 (1997), p. 352.
} 
plainte et ouvre le procès ${ }^{9}$. Admettons que Zayd ait emprunté 100 dinars à 'Amr, son voisin de Kūfa, puis soit parti s'installer à Bagdad sans rembourser la somme. Si 'Amr portait plainte auprès du cadi de sa ville, Kūfa, sa requête n'aurait en principe aucune chance d'aboutir, le cadi de Kūfa ne disposant d'aucun moyen de convoquer Zayd. La seule solution serait que 'Amr se rende à Bagdad, à environ quatre jours de distance, mais il lui resterait encore à prouver ses allégations. La principale preuve légale étant le double témoignage honorable (bayyina), il lui faudrait emmener avec lui ses témoins: une simple affaire de dette ferait donc perdre au minimum huit jours à trois personnes, sans garantie de succès puisque les témoins pourraient être récusés par le cadi de Bagdad. Pour des gens du commun liés par leurs obligations quotidiennes, de telles démarches se seraient avérées impossibles ${ }^{10}$. Or, comme l'exprime le hanafite al-Ğașșāṣ (m. 370/980), la mission du cadi consistait à « permettre aux détenteurs d'un droit d'accéder à ce droit » (iṣâal d̂awî l-ḥuqūq ilā huqūqi-him) ${ }^{11}$. Pour un faqīh, un individu résidant à l'intérieur du dār al-islām ne pouvait impunément spolier ses semblables, sans devoir craindre aucune poursuite. Il en allait probablement de même aux yeux du pouvoir, dont la légitimité reposait précisément sur sa capacité à maintenir la justice ${ }^{12}$. Il était donc juridiquement inacceptable et politiquement dangereux que la structure de la judicature entrave elle-même la réalisation de la justice. Ce problème fut résolu par le développement d'une procédure épistolaire et par la mise en place progressive d'un réseau judiciaire liant les districts les uns aux autres.

\section{Mise en place d'un réseau épistolaire}

La procédure du « kitāb al-qāậ̀ ilā l-qāộ̀ » (lettre du cadi au cadi), évoquée dans un grand nombre d'ouvrages de figh ${ }^{13}$, permit à un plaignant de poursuivre un adversaire à distance, en entamant un procès auprès du cadi de sa propre ville. À l'inverse de la procédure ordinaire, qui n'impliquait qu'un seul cadi, le procès était conduit par deux cadis successifs communiquant par voie épistolaire. Dans le cas précédemment évoqué, 'Amr portait plainte auprès du cadi de Kūfa, exposait ses griefs et produisait ses témoins. Le cadi rédigeait alors une lettre à son confrère de Bagdad, expliquant que 'Amr avait apporté devant lui la preuve de ses allégations. À réception de cette lettre, le cadi de Bagdad convoquait Zayd et le condamnait à rembourser sa dette. Dans un tel cas - probablement le plus fréquent -, la lettre du cadi expéditeur ne contenait pas de jugement mais, tel un document de tubūt, enregistrait simplement la preuve testimoniale. Dans d'autres circonstances, la missive pouvait néanmoins comporter un jugement, en particulier si le condamné s'était enfui avant l'exécution de la sentence - auquel cas le cadi destinataire était prié d'appliquer lui-

\footnotetext{
${ }^{9}$ E. Tyan, « La procédure du “défaut” en droit musulman », Studia Islamica, 7 (1957), p. 119.

${ }^{10}$ Voir les remarques d'al-Sarahsī, al-Mabsūt, Beyrouth, 1406 H., XVI, p. 95.

${ }^{11}$ Al-Ğașșāṣ, dans al-Hुașșāf, Adab al-qāộ̄, p. 237, 254. Cf. F. Ziadeh, "Compelling Defendant's Appearance at Court in Islamic Law », Islamic Law and Society, 3 (1996), p. 315.

${ }^{12}$ Voir Y. Essid, At-Tadbîr/Oikonomia. Pour une critique des origines de la pensée économique arabo-musulmane, Tunis, 1993, p. 53, 70, 86-87.

${ }^{13}$ Voir notamment: Ibn Abī Šayba, Mușannaf Ibn Abi Šayba, éd. K.Y. al-Hūt, Riyad, 1409 H., IV, p. 559 ; al-Šāfi ī, Kitāb al-umm, éd. M.Z. al-Nağğār, Beyrouth, 1973, VI, p. 211-12 ; Saḥnūn, al-Mudawwana l-kubrā, Beyrouth, s.d.,

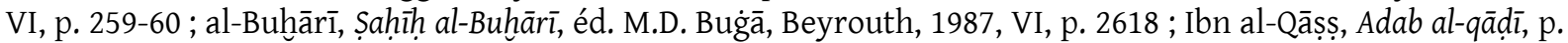
113 sq ; al-Māwardī, Adab al-qāḍ̄, éd. M.H. al-Sirḥān, Bagdad, 1971, II, p. 89 sq ; Ibn Qudāma, al-Mug̉nī, éd. 'A.-A. b. 'A.-M. al-Turkī et 'A.-F.M. al-Hulw, Riyad, 1986-90, XIV, p. 73 sq; al-Sarahssī, al-Mabsūț, XVI, p. 95 sq; alAsyūțī, Ğawāhir al-'uqūd, Beyrouth, 1996, p. 287. Voir également les références citées par W.B. Hallaq, «Qādīs communicating: legal change and the law of documentary evidence », al-Qanțara, 20 (1999), p. 439, n. 13.
} 
même le verdict - ou lorsque l'objet disputé entre deux plaideurs présents dans un même district se trouvait dans une circonscription éloignée ${ }^{14}$.

Une telle procédure était déjà mise en œuvre à l'époque umayyade : la pratique s'établit probablement dans les années $100 / 718^{15}$ et semblait répandue au début de l'époque abbasside ${ }^{16}$. C'est néanmoins dans la seconde moitié du viII ${ }^{\mathrm{e}}$ siècle que ses modalités d'applications connurent une évolution significative. Jusque-là, la correspondance entre cadis existait de fait mais ne faisait pas l'objet d'une théorisation juridique. À ce titre, l'exemple de la correspondance entre cadis est caractéristique de la formation générale des procédures judiciaires, qui furent d'abord une pratique pour n'être que par la suite théorisées par les fuqahā'. Ceux-ci se penchèrent tout particulièrement sur les modalités de rédaction, de transmission et de réception de la lettre. Seuls les deux derniers points, les plus significatifs de la formation d'un réseau judiciaire, seront ici développés.

Le mode de transmission des lettres ne fit l'objet d'aucun développement à l'époque umayyade et au début de l'époque abbasside. Quelques indices textuels permettent néanmoins d'en déduire les principales caractéristiques. Vers 101/719, le juriste kūfiote 'Umar b. Abī Zā'ida ${ }^{17}$ intenta un procès contre un débiteur résidant à Bașra et le cadi Ibn Ašwa $^{18}$ écrivit une lettre au cadi de Bașra, dans laquelle il enregistrait la bayyina en faveur du demandeur. Selon le récit d'Ibn Abī Š̀ayba, confirmé par Wak̄̄', 'Umar b. Abī Zā'ida porta lui-même la missive à son destinataire ${ }^{19}$. Vers 132/750, un bașrien nommé Ibn 'Awn entama une procédure contre un adversaire résidant à Mossoul; la lettre du cadi de Bașra fut probablement portée à celui de Mossoul par le fondé de pouvoir (wakil) du demandeur - son serviteur (g்lām) -, déjà chargé de produire ses preuves à l'audience ${ }^{20}$. Ces deux exemples montrent que la lettre était généralement transmise par le principal intéressé - le demandeur - ou son représentant, ce qui n'allait pas sans poser problème: qui d'autre qu'un plaignant avait plus intérêt à contrefaire une telle missive ? En tout état de cause, la correspondance entre cadis était portée par des particuliers, comme semble encore le confirmer l'envoi d'une lettre d'Abū Šayba (cadi de Wāsiț, m. 169/785-86 ${ }^{21}$ ) à Ibn Abī Laylā par l'intermédiaire d'un individu identifié comme le père de Sa'īd b. Yahyā Abū Sufyān alHimyarī $^{22}$. La correspondance judiciaire n'empruntait pas un réseau étatique déjà constitué tel celui du barīd, auquel on eût pourtant pu supposer un rôle compte tenu des hautes fonctions officielles exercées par les cadis ${ }^{23}$. Peut-être leurs lettres n'étaient-elles pas considérées comme assez importantes pour la sûreté de l'État ${ }^{24}$; peut-être eût-il également

\footnotetext{
${ }^{14}$ Ibn al-Qāșṣ, Adab al-qāḍ̄, p. 113. Sur cette procédure, voir E. Tyan, Le notariat et le régime de la preuve par écrit dans la pratique du droit musulman, Beyrouth, 1945, p. 87 ; J. Schacht, Introduction au droit musulman, Paris, 1983, p. 160 ; Hallaq, art. cit., p. 437-66 ; P. Scholz, « Legal Practice in the Malikite Law of Procedure », al-Qantara, 20 (1999), p. 424-25.

${ }^{15}$ W.B. Hallaq, The Origins and Evolution of Islamic Law, Cambridge, 2005, p. 61.

${ }^{16}$ Wakī' Ahbār al-quḍāt, éd. 'A.-A.M. al-Marāḡì, Le Caire, 1947-50, II, p. 49-50, 52, 119 ; III, p. 255.

${ }^{17}$ Voir Ibn Hağar, Lisān al-Mīzān, Beyrouth, 1986, VII, p. 317.

${ }^{18}$ Sur ce cadi, voir Wakī̄ Ahbār al-qud̄àt, III, p. 10-21.

${ }^{19}$ Ibn Abī Šayba, Mușannaf Ibn Abī Šayba, IV, p. 559 ; Wakīì Ahbār al-quḍ̄at, II, p. 11.

${ }^{20}$ Wakî̀, Ahbār al-quḍāt, II, p. 49-50. Il apparaît qu'Ibn 'Awn ne porta pas lui-même la lettre, car le cadi de Mossoul, qui voulait lui faire prêter serment, dut à son tour écrire au cadi de Bașra. Ce dernier répondit que le demandeur refusait de jurer.

${ }^{21}$ Al-Hुațīb, Ta'rīh Madìnat al-salām, éd. B.'A. Ma'rūf, Beyrouth, 2001, VII, p. 21-26.

${ }^{22}$ Wakī', Ahbār al-qud̄āt, III, p. 310. Sur ce savant de Wāsiț, m. 202/818, voir Ibn Sa'd, al-Ṭabaqāt al-kubrā, Beyrouth, 1968, VI, p. 314 ; al-Dahabī, Siyar a'ām al-nubalā', éd. Š. al-Arnā'ūț et M.N. al-'Arqasūsī, Beyrouth, 1413 H., IX, p. 432. Un autre cas de lettre portée par le demandeur est mentionné par Wakî̀, Ahbār al-quḍāt, III, p. 311.

${ }^{23} \mathrm{Au}$ début de l'époque abbasside, le barīd acheminait les candidats à la judicature vers Bagdad et les cadis vers leurs nouveaux postes. Wakî̀, Ahbār al-qudāt, II, p. 122, 165.

${ }^{24}$ Sur les fonctions du barīd au début de l'époque abbasside, voir Silverstein, op. cit., p. 78 sq.
} 
paru incohérent d'acheminer une lettre par un service rapide quand le principale intéressé, le demandeur ou son wakil, devait se rendre par ses propres moyens - et beaucoup plus lentement - auprès du cadi destinataire pour être confronté à son adversaire. Enfin, le réseau du barīd était probablement inadapté à la communication entre cadis : jusqu'au milieu $\mathrm{du} \mathrm{III}^{\mathrm{e}} / \mathrm{IX}^{\mathrm{e}}$ siècle, la poste officielle fut une institution aux structures malléables, modifiées par chaque calife selon la situation de son époque ${ }^{25}$; de surcroît, le barīd avait pour fonction principale de relier les villes provinciales à la capitale, alors que les cadis s'écrivaient surtout de province à province.

La réception de la lettre fut quant à elle le premier élément à faire l'objet d'une réflexion juridique. Phase décisive avant le rendu ou l'application du jugement, elle constituait aussi le moment le plus critique, où le destinataire devait décider de l'authenticité de la missive. La sécurité du réseau épistolaire apparut d'abord comme étroitement liée à cette ultime étape. En raison de la gravité des informations échangées, conduisant à la condamnation d'un individu sans audition directe des preuves, le destinataire devait être absolument certain de l'origine de la lettre. Les cadis furent les premiers à réfléchir aux meilleurs moyens d'assurer une communication fiable avec leurs homologues. Au début de l'époque abbasside, l'authentification d'une lettre reposait essentiellement sur l'observation et l'identification de son sceau (hātam). Chaque cadi possédait un sceau en propre, qu'il utilisait non seulement pour cacheter sa correspondance et sceller ses archives, mais également pour certifier l'authenticité d'un bien en litige transporté entre deux districts ou l'origine d'une convocation au tribunal ${ }^{26}$. Au commencement du II $^{\mathrm{e}} / \mathrm{VIII}^{\mathrm{e}}$ siècle, le célèbre cadi de Kūfa 'A/mir al-Ša'bī (m. entre 103/721 et 110/728) acceptait la lettre d'un confrère à la simple condition qu'elle fût cachetée ${ }^{27}$. Au début de sa carrière judiciaire, Ibn Abī Laylā (cadi de Kūfa de 132/750 à 148/765-766) entérinait encore les missives de ses confrères à la seule vue de leurs sceaux. Il observait avec soin le cachet imprimé sur la lettre, sortait éventuellement l'examiner à la lumière du jour et tirait légèrement dessus pour vérifier qu'il ne se détachait pas trop facilement ${ }^{28}$.

L'identification d'une lettre de cadi par ce biais nécessitait néanmoins la connaissance préalable de son sceau. Or, compte tenu de l'immensité du dār al-islām et du rythme soutenu des désignations et des révocations à la judicature, cette connaissance était forcément limitée. Peut-être les réseaux judiciaires étaient-ils assez réduits à l'époque umayyade pour que la simple observation du sceau ait suffi à établir l'origine d'une missive. Mais au début de l'époque abbasside, la communication entre districts avait pris une telle ampleur qu'un cadi scrupuleux ne pouvait plus s'en tenir à cela. Admettant qu'un cadi pouvait fort bien ne pas reconnaître le sceau d'un de ses homologues, Ibn Abī Laylā se mit à préconiser, en pareil cas, la production d'une preuve testimoniale (bayyina): si le sceau ne pouvait être authentifié par le cadi destinataire, deux témoins devaient le reconnaitre pour lui ${ }^{29}$. À peu près à la même époque, le cadi bașrien Sawwār b. 'Abd Allāh (140/757-58 à 156/773) adopta la même méthode ${ }^{30}$. Jusque-là, la matérialité de l'écrit lui donnait une valeur probante ${ }^{31}$.

\footnotetext{
${ }^{25}$ Ibid., p. 88.

${ }^{26}$ Al-Hașșāf, Adab al-qāḍ̄, p. 245, 335, 415. Un exemple d'inscription figurant sur un sceau de cadi est donné par Wakî̀, Ahbār al-qudāt, III, p. 18.

${ }^{27}$ Ibn Abī Šayba, Mușannaf Ibn Abī Šayba, IV, p. 559 ; Wakī̄, Aḩbār al-quḍāt, II, p. 416. Sur 'A/mir al-Ša'bī, voir G.H.A. Juynboll, « al-Sha'bī », $E I^{2}$, IX, p. 162-63.

${ }^{28}$ Wakî̀, Ahbār al-quḍāt, III, p. 133. Principal représentant d'un courant juridique kūfiote antérieur à la formation des écoles juridiques « personnelles », et plus tard intégré rétroactivement dans le madhab hanafite, Ibn Abī Laylā est connu pour s'être en grande partie fondé sur son expérience judiciaire dans l'élaboration de sa doctrine. J. Schacht, « Ibn Abī Laylā »,EI², III, p. 709.

${ }^{29}$ Wakî̀, Ahbār al-quḍāt, II, p. 67 ; III, p. 134.

${ }^{30}$ Al-Buhārī, Șahịḥ al-Buhārī, VI, p. 2618.
} 
Avec l'extension du réseau épistolaire, cette valeur déclina: tout signe matériel de la provenance de la lettre apparaîtrait bientôt comme secondaire, voire superflu. C'est que, parallèlement, les modes d'acheminement de la lettre avaient considérablement évolué et garantissaient - mieux que le sceau - l'authenticité du message.

Les caractéristiques de la procédure épistolaire au cours de sa période formative conduisent à formuler deux conclusions. Tout d'abord, la principale institution judiciaire n'utilisait pas un réseau de communication étatique pour l'acheminement de son courrier officiel. L'État était présent aux deux extrémités du parcours - à travers les cadis émetteurs et récepteurs -, mais le transport des lettres était à la charge d'un particulier extérieur à l'administration judiciaire. En second lieu, le pouvoir délégant (le gouverneur, puis le calife) ne réglementait nullement cette procédure et laissait à ses cadis la liberté de créer leurs réseaux, d'en élaborer le fonctionnement et de les faire vivre par leurs propres moyens. Bien que l'«État» fût une réalité depuis le début de l'époque umayyade, à travers un ensemble cohérent d'institutions ${ }^{32}$, il apparaissait ici comme un État minimaliste, peu soucieux de réglementer le fonctionnement interne de la judicature ni de coordonner l'action de la justice à l'échelle impériale. Se considérant comme les représentants de Dieu sur terre ${ }^{33}$, les califes umayyades et les premiers Abbassides se permettaient d'intervenir dans les procès pour orienter ou casser le jugement des cadis ${ }^{34}$. Mais derrière les manifestations épisodiques de cet autocratisme califien ${ }^{35}$, l'État islamique laissait un vide institutionnel que des juristes privés entreprirent de combler.

\section{L'institutionnalisation du réseau judiciaire}

Les procédures de réception de la lettre, si sophistiquées qu'elles fussent, ne réduisaient pas à néant les risques de fraude et de contrefaçon. Le premier danger résidait dans l'absence de confrontation entre le défendeur et les témoins qui avaient déposé contre lui. Wakī rapporte que le cadi de Wāsiț, Abū Šayba, reçut un jour une lettre de son homologue kūfiote par l'intermédiaire d'un demandeur. Il convoqua le défendeur, nommé al-Ḥağğăğ b. Dīnār, contre qui le cadi de Kūfa avait enregistré une preuve testimoniale. Surpris par la teneur de l'accusation, le défendeur protesta de son innocence et demanda un ajournement de l'audience. Il profita du délai qui lui était accordé pour se rendre à Kūfa et, sous un prétexte fallacieux, fit convoquer par le cadi le témoin qui avait déposé contre lui. La confrontation permit d'établir que le témoin n'avait en réalité jamais rencontré al-Hağğgăğ b. Dīnār et qu'il avait été adroitement manipulé par le demandeur. Le cadi de Kūfa écrivit donc une nouvelle lettre à celui de Wāsit pour annuler sa première missive et les poursuites contre al-Hağğăğ b. Dīnār furent abandonnées ${ }^{36}$. Grâce au voyage que le défendeur put entreprendre, le complot avorta. Mais tout accusé ne pouvait probablement abandonner ainsi ses affaires pour un long voyage et les risques d'erreurs judiciaires dues à la malveillance d'un habile plaideur demeuraient grands.

\footnotetext{
${ }^{31}$ Ibn Abī Laylā, dont la doctrine était représentative de l'ancien courant kūfiote, accordait une valeur beaucoup plus grande à l'écrit (notamment aux archives judiciaires) que les Ḥanafites. Voir Abū Yūsuf, Ihtilāf Abī Hanīfa wa-Ibn Abī Laylā, éd. Abū al-Wafā' et R.M. Riḍ̂ān, Hayḍarābād, 1357 H., p. 158-59.

${ }^{32}$ F.M. Donner, « The Formation of the Islamic State », Journal of the American Oriental Society, 106 (1986), p. 28396.

${ }^{33}$ P. Crone et M. Hinds, God's Caliph, Cambridge, 1986, p. 46 et passim ; P. Crone, God's Rule, New York, 2004, p. 130 ; W. al-Qādī, « The Religious Foundation of Late Umayyad Ideology », dans Saber religioso y poder político en el Islam, Madrid, 1994, p. 250.

${ }^{34}$ Voir M. Tillier, « Un traité politique du II $/$ VIII $^{e}$ siècle. L'épître de 'Ubayd Allāh b. al-Ḥasan al-'Anbarī au calife al-Mahd̄̄ », Annales Islamologiques, 40 (2006), p. 143-44.

${ }^{35} \mathrm{Cf}$. Silverstein, op. cit., p. 88.

${ }^{36}$ Wakî̀, Ahbār al-qudāt, III, p. 311.
} 
Les erreurs ou les fraudes lors de l'acheminement de la lettre représentaient un second danger. Une lettre pouvait être contrefaite et portée par un plaideur qui, en réalité, n'avait jamais eu affaire à un quelconque cadi expéditeur, ou bien une lettre authentique pouvait être subtilisée et remplacée en cours de route ${ }^{37}$. Conscients des faiblesses de la procédure épistolaire, les juristes musulmans réfléchirent au moyen de sécuriser la transmission de la lettre. Au milieu du $\mathrm{III}^{\mathrm{e}} / \mathrm{IX}^{\mathrm{e}}$ siècle, le hanafite al-Hुașșāf insista tout d'abord sur les règles de rédaction d'une telle missive. Le défendeur devait y être nominalement identifié avec une précision telle que tout risque d'homonymie soit écarté ; si toutefois un doute demeurait sur l'identité de l'accusé, la procédure devait être interrompue. La date mentionnée dans la lettre permettait éventuellement de dissiper les doutes à ce sujet, en écartant de l'accusation les homonymes décédés avant la rédaction. De même, l'identité et l'adresse des témoins de l'affaire devaient être soigneusement enregistrées dans la missive, afin que le cadi destinataire puisse diligenter une enquête à leur sujet ${ }^{38}$. Mais, surtout, les fuqaha $\bar{a}$ tentèrent de sécuriser le transport de la lettre. Au II ${ }^{\mathrm{e}} / \mathrm{VIII}^{\mathrm{e}}$ siècle, Ibn Abī Laylā et Sawwār b. 'Abd Allāh avaient entrepris d'appliquer le principe de la bayyina à la procédure épistolaire, en faisant authentifier l'objet-lettre par deux témoins qui en reconnaissaient le sceau. Peutêtre prévoyaient-ils déjà que ces témoins accompagnent le demandeur. Mais une telle authentification n'écartait pas tout risque de fraude: al-Šăfiī évoque une affaire où une lettre apparemment authentique dans son aspect extérieur et portée en compagnie de deux témoins s'avéra finalement contrefaite ${ }^{39}$. C'est pourquoi les juristes hanafites prescrivirent finalement que la lettre soit accompagnée par deux témoins honorables qui ne témoigneraient pas simplement de l'authenticité matérielle de la missive, mais également de son contenu. Al-Hुașsâaf demande au cadi expéditeur de lire sa lettre devant les témoins avant de la fermer et de leur en donner à chacun une copie leur servant d'aide-mémoire (tadkira $)^{40}$. Pour des cadis ḥanafites comme al-Ḥasan b. Ziyād al-Lu'lu'ī (m. 204/819-20), leur confirmation orale du contenu de la lettre était essentielle : ils devaient donc la connaitre par cœur ou, à défaut, en posséder une copie ${ }^{41}$. Dans ces conditions, l'authentification matérielle de la lettre passait au second plan : le message pouvait être accepté même si le sceau du cadi expéditeur était brisé avant son arrivée à destination ${ }^{42}$.

Tous les juristes hanafites ne considéraient pas le témoignage du contenu de la lettre comme indispensable ${ }^{43}$. Le courant se réclamant d'Abū Yūsuf demeurait attaché à la forme de la lettre : les témoins devaient surtout attester de sa provenance et de l'authenticité du sceau $^{44}$. Il n'en demeure pas moins que, dans bien des cas, l'acheminement de la lettre en fut considérablement transformé : elle n'était plus confiée au seul demandeur ou à son représentant, mais convoyée par deux témoins qui, garants de son contenu, devenaient par là même les véritable porteurs de la missive. En confiant sa parole écrite à des témoins désignés pour cette mission, le cadi institutionnalisait le transport de la lettre. Cette procédure relevait juridiquement d'une extension de la bayyina, la preuve reine, à la correspondance entre cadis. Mais comment ne pas souligner la similitude entre cette procédure et d'antiques modes de communication? Dans l'Arabie préislamique, les messages importants étaient souvent confiés à deux courriers, afin de doubler leurs chances

\footnotetext{
${ }^{37}$ Al-Šāii ī, Kitāb al-umm, VI, p. 211.

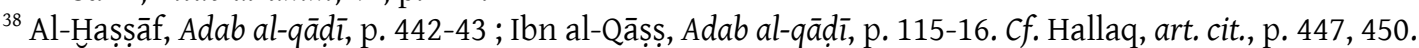

${ }^{39}$ Al-Šăfi ${ }^{i}$, Kitāb al-umm, VI, p. 211.

${ }^{40}$ Al-Hुașșāf, Adab al-qāệ̀, p. 410. Cf. al-Māwardī, Adab al-qāḍ̄i, II, p. 130.

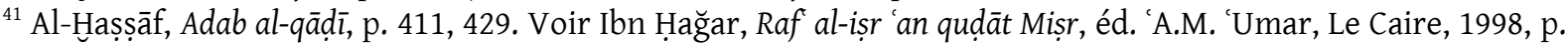
290 (trad. M. Tillier, Vies des cadis de Mișr, Le Caire, 2002, p. 162).

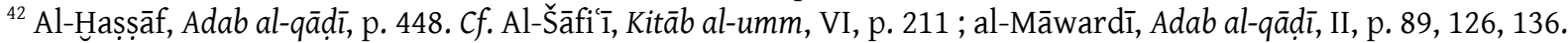
Voir Hallaq, art. cit., p. 451 ; Johansen, « Formes de langage », p. 355.

${ }^{43}$ Johansen, « Formes de langage », p. 357.

${ }^{44}$ Al-Ḩașsāâf, Adab al-qā ḍì p. 411.
} 
de délivrance ${ }^{45}$ - et, éventuellement, d'en garantir l'authenticité. Cet usage avait perduré à l'époque islamique et peut-être l'évolution de la procédure épistolaire fut-elle aussi influencée par cette ancienne coutume arabe.

On pourrait croire en apparence que l'introduction du témoignage institutionnel dans la procédure épistolaire contredisait son objectif premier : éviter aux témoins d'une affaire d'accompagner le demandeur dans son voyage. Ce n'était pourtant pas le cas. En effet les témoins envoyés avec la missive n'étaient pas ceux du litige. Ces derniers se contentaient de leur déposition devant le cadi expéditeur, puis retournaient à leurs activités quotidiennes sédentaires. À l'inverse, les témoins de la lettre étaient désignés pour cette seule mission. Encore reste-t-il à déterminer qui étaient ces témoins susceptibles de s'absenter pendant de longues périodes afin de convoyer la correspondance judiciaire. Deux hypothèses peuvent être envisagées : il s'agissait soit de témoins instrumentaires, soit de témoins occasionnels. En Iraq, ces deux possibilités ne s'excluent pas mutuellement et correspondent peut-être à deux étapes successives.

L'apparition de témoins instrumentaires est traditionnellement associée à celle de listes officielles de šuhūd, au tournant $\mathrm{du} \mathrm{II}^{\mathrm{e}} / \mathrm{VIIII}^{\mathrm{e}}$ et $\mathrm{du} \mathrm{III}^{\mathrm{e}} / \mathrm{IX}^{\mathrm{e}}$ siècle ${ }^{46}$. Dans une logique plus large de renforcement des structures judiciaires, les tribunaux intégrèrent de manière durable des témoins à l'honorabilité bien établie ${ }^{47}$. Les historiens de l'Islam considèrent souvent que l'institution des témoins instrumentaires professionnels se généralisa dès le début du $\mathrm{III}^{\mathrm{e}} / \mathrm{IX}^{\mathrm{e}}$ siècle. Le cadi s'entourait d'un nombre limité de témoins triés sur le volet, que les particuliers sollicitaient pour valider leurs actes et, éventuellement, témoigner en justice. C'est à eux, de même, que le cadi pourrait avoir confié sa correspondance. Néanmoins, comme le soulignait Claude Cahen en 1970, rien ne prouve que la judicature suivit une évolution similaire dans toutes les provinces du dār al-islām. En Égypte, la formation d'un groupe de témoins instrumentaires autour du cadi est attestée à une époque ancienne, grâce aux biographies d'al-Kindī. Mais en Iraq, les Ahbār al-qud̄āt de Wakî ne recèlent pas trace de quelconques listes de šuhūd et Cahen propose que l'émergence d'un corps restreint de témoins instrumentaires ne s'y produisit qu'au $\mathrm{IV}^{\mathrm{e}} / \mathrm{X}^{\mathrm{e}}$ siècle, par contamination du modèle égyptien ${ }^{48}$.

Cette supposition n'est pas à prendre à la légère. L'Adab al-qādì d'al-H̦așsāa représentatif de la doctrine iraqienne et inédit lorsque Cahen écrivit son article - en confirme peut-être la pertinence. Les passages attribuables à al-Hुașsāa lui-même - et non à son commentateur al-Ğașsāạ - ne font nulle référence à un groupe exclusif de témoins instrumentaires et présupposent, conformément à la doctrine d'Abū Ḥanīfa, que tout bon musulman est susceptible de témoigner ${ }^{49}$. Cela ne signifie pas, bien sûr, que les cadis iraqiens n'employaient pas des hommes honorables pour les assister à l'audience. Al-Hasșsāaf signale ainsi que la lettre d'un cadi peut porter les sceaux de témoins à côté du sien ${ }^{50}$, ce qui laisse penser qu'au milieu du $\mathrm{III}^{\mathrm{e}} / \mathrm{IX}^{e}$ siècle, des témoins étaient déjà sélectionnés par les cadis iraqiens pour travailler à leurs côtés. En revanche, un siècle plus tard, al-Ğașșāṣ manifeste une tendance certaine à interpréter le texte de son prédécesseur dans le sens d'une institutionnalisation stricte du témoignage. Lors de l'arrivée en poste d'un cadi, al-

\footnotetext{
${ }^{45}$ Silverstein, op. cit., p. 45-46.

${ }^{46} \mathrm{Cl}$. Cahen, « À propos des shuhūd », Studia Islamica, 31 (1970), p. 75.

${ }^{47}$ Pour les détails de ces transformations, voir E. Tyan, Histoire de l'organisation judiciaire en pays d'islam, Leyde, 1960, p. 238-42; B. Johansen, "Wahrheit und Geltungsanspruch: zur Begründung und Begrenzung der Autorität des Qadi-Urteils im islamischen Recht », dans O. Capitani et alii, La Giustizia nell'alto medioevo (secoli IXXI), Spoleto, 1997, p. 1006-1012.

${ }^{48}$ Cahen, «À propos des shuhūd », p. 76.

${ }^{49}$ Al-Hașșāf, Adab al-qādì̄, p. 289 sq.

${ }^{50}$ Al-Hुașșāf, Adab al-qā dị̄, p. 448.
} 
Hașsāaf lui demande de se renseigner sur les hommes honorables ('udūl) et fiables (ahl al-țiqa wa-l-amāna) de la ville et d'enregistrer leurs noms par quartiers. Avec le recul, cela pourrait passer pour l'établissement d'une liste de témoins instrumentaires. Pourtant, l'auteur ne parle pas explicitement de šuhūd dans ce passage, ni ne semble employer le terme 'udūl dans le sens restrictif de "[témoins] honorables ». Al-Hașșāf porte visiblement l'accent sur la «fiabilité » des individus ainsi répertoriés, ce qui sous-entend qu'il ne souhaite pas spécifiquement les utiliser comme témoins, mais plutôt comme "hommes de confiance " (umanä') chargés d'administrer des biens pour le cadi. C'est seulement dans le commentaire d'al-Ğașșāṣ que les deux catégories sont assimilées et que le terme šuhūd fait son apparition : pour le juriste $\mathrm{du} \mathrm{IV}^{\mathrm{e}} / \mathrm{x}^{\mathrm{e}}$ siècle, cette enquête préliminaire entend classer les habitants en catégories sociales hiérarchisées (yurattibu li-kull wāhid min-hum martaba 'alā mā yastahiqquhu wa-yunazzilu-hum manāzila-hum), procédure caractéristique de la définition d'une élite de témoins instrumentaires ${ }^{51}$.

C'est donc au cours du siècle qui sépare al-Hasșââ d'al-Ğașsạsạ que la restriction du témoignage à un groupe social restreint sembla se produire en Iraq. Un récit d'al-Tanūhī, déjà remarqué par Claude Cahen, vient étayer cette hypothèse. Ayant entendu qu'un ancien cadi de Bașra, al-Taymi ${ }^{52}$, avait agréé 36000 témoins lors de sa judicature, al-Tanūh̆i fut fort étonné et demanda des explications. Il lui fut répondu que tout individu honorable pouvait alors témoigner en faveur de son voisin et « qu'on ne connaissait pas de classement (tartib) [des individu] en un groupe spécialisé dans le témoignage » : cette situation aurait perduré jusqu'à Ismā̄îl [b. Ishāā], cadi mālikite de Bagdad à partir des années $250 / 864^{53}$. Si cela est exact, c'est donc vers la fin de la vie d'al-Haș̦ââf - peut-être à l'époque même où il rédigea son traité - que le témoignage commença, peu à peu, à se restreindre (de façon non exclusive ${ }^{54}$ ) à un groupe d'élite.

On peut donc envisager deux phases successives dans le renforcement de la procédure épistolaire :

1. $A u \mathrm{III}^{\mathrm{e}} / \mathrm{IX}^{\mathrm{e}}$ siècle, les cadis iraqiens commençaient peut-être à prendre, parmi leurs auxiliaires, quelques témoins instrumentaires, mais il n'existait pas encore de groupe social limité de šuhūd. Le témoignage demeurait majoritairement le fait de témoins occasionnels, agréés de manière circonstancielle. À qui un cadi demandait-il donc d'accompagner ses lettres? Aux quelques témoins dont il s'entourait ? Cela est peu vraisemblable, car l'activité du tribunal aurait souffert de leur éloignement. À des témoins occasionnels, connus pour leur honorabilité et chargés de missions ponctuelles? Cela semble plus probable, bien que demeure la question de leur rémunération. Étaient-ils payés par le cadi? Le cadi bașrien Sawwār b. 'Abd Allāh rémunérait les hommes de confiance à qui il confiait l'administration de biens (umanā') ${ }^{55}$. Peut-être étaient-ils payés par les demandeurs - auquel cas cela marquerait le début de la «professionnalisation » du notariat en Iraq -, mais les sources n'en gardent pas trace. Enfin, si le modèle du témoignage occasionnel et non-professionnel

\footnotetext{
${ }^{51}$ Al-Hașșāf et al-Ğașṣāṣ, dans al-Ḩașsāf, Adab al-qāḍ̄, p. 83-84.

${ }^{52}$ Plusieurs personnages portant la nisba « al-Taymī » exercèrent la judicature de Bașra à l'époque abbasside : 'Umar b. 'Uțmān al-Taymī, sous al-Mahdī ou al-Rašìd; Ibrāhīm b. Muhammad al-Taymī, de 239/853-54 à 247/861-62 ; Ahmad b. 'Abd al-Karīm, vers 247-248/861-63. Voir M. Tillier, Les cadis d'Iraq à l'époque 'abbāside, p. $625-26$.

${ }^{53}$ Al-Tanūhn̄ī, Nišwār al-muhāẹdara, éd. 'A. al-šālğ̄i, s.l., 1971, I, p. 140.

${ }^{54}$ Dans un passage sur la définition des confins d'une propriété en litige, al-Ğașșāṣ distingue nettement les témoins occasionnels d'une catégorie de témoins instrumentaires au service du cadi (al-Ğașșāș, dans al-Haș̣̦āf, Adab al-qādì , p. 386). Cela suggère qu'à la fin $\mathrm{du} \mathrm{IV}^{\mathrm{e}} / \mathrm{x}^{\mathrm{e}}$ siècle, la parole de témoins occasionnels pouvait encore être prise en considération dans la procédure judiciaire.

${ }^{55}$ Wakî̀, Ahbār al-quḍăt, II, p. 58. W.B. Hallaq (The Origins and Evolution, p. 88) interprète ce passage comme la preuve de l'existence, dès la fin $\mathrm{du}_{\mathrm{II}}^{\mathrm{e}} / \mathrm{VIII}^{\mathrm{e}}$ siècle, d'un corps de témoins instrumentaires salariés par le cadi. Pourtant le texte de Wakî̀ ne fait allusion qu'aux umanā', et non aux témoins.
} 
était encore dominant, pourquoi ne pas envisager que la correspondance entre cadis ait emprunté des réseaux extrajudiciaires préexistants? A.J. Silverstein a montré le rôle essentiel des marchands, dès le $\mathrm{III}^{\mathrm{e}} / \mathrm{IX}^{\mathrm{e}}$ siècle, pour la circulation des nouvelles officielles. Leurs lettres étaient parfois lues devant des cadis, qui transmettaient les informations importantes au pouvoir politique ${ }^{56}$. Qui, mieux que de tels professionnels du voyage, pouvait aisément convoyer une lettre de cadi en compagnie du demandeur? Il suffisait que deux marchands, agréés comme témoins, soient en partance pour la ville du destinataire, pour que le cadi expéditeur recourre à leur service - peut-être bénévole. Ce n'est là qu'une conjecture, mais qui ne semble pas invraisemblable. Le réseau judiciaire fonctionnerait alors en partenariat avec des réseaux privés. Il s'agirait donc d'un réseau mixte : étatique par son rattachement, aux deux extrémités, à l'institution judiciaire, mais privé dans ses modes d'acheminement.

2. Au $\mathrm{IV}^{\mathrm{e}} / \mathrm{X}^{\mathrm{e}}$ siècle, le modèle égyptien se serait généralisé en Iraq et une classe réduite de témoins instrumentaires sélectionnés par le cadi aurait accaparé la majorité des témoignages. Il est probable que l'accompagnement des lettres judiciaires leur soit alors également échu. L'utilisation de témoins professionnels attachés aux tribunaux témoignerait de la mise en place d'un réseau étatique fort : l'institution judiciaire assurerait désormais le transport de la lettre, le demandeur n'ayant plus à se déplacer qu'en raison de sa nécessaire comparution devant le cadi destinataire. En cas de longue distance entre deux circonscriptions, un système de relais fut envisagé : en autorisant le témoignage rapporté (al-šahāda 'alā l-šahāda), les juristes permirent aux témoins initiaux de transmettre leur déposition à d'autres témoins ${ }^{57}$. Si le cadi de Kūfa écrivait à celui de Hamadān, ses témoins pouvaient accompagner la lettre jusqu'à Bașra, y passer le relais à deux témoins locaux, puis retourner chez eux. En théorie, une chaîne de témoins instrumentaires reliait donc entre eux tous les districts de l'empire, permettant la circulation d'une lettre judiciaire d'un bout à l'autre du monde musulman. De même, si le demandeur découvrait, à son arrivée à Hamadān, que son adversaire avait entre temps déménagé à Marw, il pouvait réclamer une nouvelle lettre adressée au cadi de cette dernière localité et poursuivre son voyage ${ }^{58}$.

Il faut néanmoins nuancer le caractère "étatique» d'un tel réseau. Les témoins «professionnels» étaient certes sélectionnés par le cadi, placés sous son autorité hiérarchique ${ }^{59}$ et enregistrés en nombre limité auprès du tribunal, mais ne semblaient pas rémunérés par ce dernier ni par le Trésor public: plus probablement payés par les particuliers recourant à leurs services, ils constituaient certes des "notaires " liés à l'autorité souveraine, mais fonctionnaient au quotidien comme une institution privée. Il est possible que les témoins instrumentaires choisis par le cadi pour acheminer sa correspondance aient été payés par le tribunal, comme d'autres auxiliaires, mais nous n'en avons pas trouvé de preuve textuelle. Par ailleurs, l'organisation des réseaux épistolaires relevait des seuls cadis, qui se fondaient sur la réflexion des fuqahä' et non sur des instructions officielles de l'administration centrale. Dans un des rares modèles d'actes d'investiture connus pour l'époque abbasside - reproduit par Qudāma b. Ğa'far -, le calife ordonne certes à son cadi d'accepter les missives de ses homologues, mais ses consignes demeurent très générales et n'abordent nullement les moyens d'acheminement de telles lettres. Leur authenticité semble encore devoir être établie par vérification du sceau et

\footnotetext{
${ }^{56}$ Silverstein, op. cit., p. 117-19.

${ }^{57}$ Al-Hुașsāâ, Adab al-qāậ̀, p. 436. Al-Šaybānī autorise le témoignage rapporté si le témoin original est malade ou se trouve à plus de trois jours de l'audience. Al-Šaybānī, al-Ğāmi al-ṣagìr, Beyrouth, 1986, p. 392 ; Ibn al-Qāșs, Adab al-qādīi, p. 107 sq. Sur le témoignage rapporté, voir Schacht, Introduction, p. 161; R. Brunschvig, Études d'islamologie, Paris, 1976, II, p. 214.

${ }^{58}$ Al-Hुașșāf, Adab al-qāộ̀, p. 435.

${ }^{59}$ Tyan, Organisation judiciaire, p. 250.
} 
l'utilisation de témoins pour accompagner la lettre n'apparaît pas ${ }^{60}$. Au IV $/ \mathrm{X}^{\mathrm{e}}$ siècle, la procédure épistolaire est ainsi représentative du fonctionnement plus général de la judicature, sorte de coquille étatique réglementée de l'intérieur par la réflexion combinée de juristes privés et de cadis.

\section{Conclusion}

Sans la procédure épistolaire, il eût été presque impossible pour un demandeur de prouver ses allégations dans une circonscription étrangère au préjudice. Bien plus, l'empire islamique se serait présenté comme une juxtaposition de districts indépendants les uns des autres, seulement reliés au pouvoir par le jeu administratif des désignations et des révocations. Même si les précautions peu à peu élaborées pour sécuriser la procédure n'écartaient pas tout risque, le réseau scripturaire reliant les cadis entre eux apparait, en fin de compte, comme le principal moyen de réaliser l'unité judiciaire voulue par les Abbassides.

Il convient pourtant de souligner les limites d'un tel réseau, dues en grande partie aux contraintes d'une procédure longue et aléatoire. Non seulement les témoins du litige devaient être agréés avant que la lettre ne soit expédiée ${ }^{61}$, mais certains juristes préconisaient aussi que le cadi destinataire diligente une enquête d'honorabilité sur les témoins de la missive ${ }^{62}$. Cela supposait plusieurs allers-retours entre les districts du destinataire et de l'expéditeur. Après avoir reçu une lettre de son homologue de Kūfa, le cadi de Bagdad envoyait des auxiliaires à Kūfa, qui enquêtaient sur les témoins puis revenaient à Bagdad. Alors, seulement, le cadi destinataire ouvrait la lettre et menait le procès à son terme. Si les deux districts étaient plus éloignés encore, la procédure pouvait durer des semaines, voire des mois; l'enquête devenait un véritable casse-tête lorsque la lettre était identifiée grâce à un témoignage rapporté. On peut douter que les cadis aient disposé d'un personnel assez nombreux pour respecter rigoureusement des règles aussi contraignantes. Dans quelle mesure la procédure épistolaire était-elle d'ailleurs employée sur la longue distance? Pour les juristes hanafites, le cadi destinataire ne pouvait accepter la lettre qu'à condition que le cadi expéditeur fût encore en poste au moment de la réception ${ }^{63}$. En recourant à cette procédure, un plaideur prenait donc le risque d'effectuer le voyage en vain : si le cadi expéditeur mourait ou se voyait révoqué avant son arrivée à destination, et si le cadi destinataire en apprenait la nouvelle, il n'ouvrirait même pas la lettre. Dans la partie majoritairement hanafite de l'empire ${ }^{64}$, il est donc possible que les communications entre cadis aient été géographiquement réduites à une province ou une série de provinces limitrophes.

Dans un ouvrage récent, Benjamin Jokisch a proposé que, loin de surgir d'une réflexion indépendante, la constitution du fiqh préclassique procéda d'une codification étatique, à l'époque de Hārūn al-Rašīd, par l'adaptation du droit romain précédemment codifié dans l'Empire byzantin ${ }^{65}$. Cette hypothèse simplifie considérablement la compréhension de la judicature, dont le fonctionnement pourrait simplement procéder d'une telle entreprise étatique. Néanmoins, la formation du réseau judiciaire épistolaire reflète une histoire bien différente. La correspondance entre cadis apparaît avant tout comme une pratique,

\footnotetext{
${ }^{60}$ Qudāma b. Ğa far, Kitāb al-ȟarāăg, p. 43.

${ }^{61}$ Hallaq, art. cit., p. 445-46.

${ }^{62}$ Al-Hुașșāf, Adab al-qāḍī, p. 433. Cf. Ibn al-Qāṣș, Adab al-qāḍ̄, p. 119.

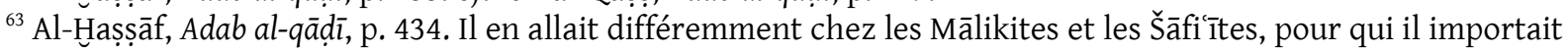
seulement que le cadi ait été en poste au moment de l'expédition. Hallaq, art. cit., p. 452.

${ }^{64}$ Sur cet espace, voir N. Tsafrir, The History of an Islamic School of Law. The Early Spread of Hanafism, Cambridge, 2004.

${ }^{65}$ B. Jokisch, Islamic Imperial Law. Harun-Al-Rashid's Codification Project, Berlin, 2007, p. 279 sq.
} 
enracinée dans l'époque umayyade, qui se perfectionna au gré de la réflexion personnelle des cadis eux-mêmes. Cette pratique fit ensuite l'objet d'une théorisation juridique mais en marge de l'État, par des fuqahā' indépendants. De son côté, le pouvoir politique n'entreprit nullement de codifier la procédure. Les instructions qu'il envoyait à ses cadis lors de leur investiture étaient à peine allusives ; bien plus, si le modèle reproduit par Qudāma b. Ğa far date bien $\mathrm{du} \mathrm{IV} / \mathrm{x}^{\mathrm{e}}$ siècle, ces recommandations accusaient un retard sérieux sur la réflexion juridique et sur la pratique elle-même. Le figh ne se développa pas seulement, à ses débuts, comme une critique de la pratique umayyade ${ }^{66}$ : il vint également combler un vide dans un secteur normatif largement laissé à l'abandon par le pouvoir califal.

${ }^{66} \mathrm{~J}$. Schacht, The Origins of Muhammadan Jurisprudence, Oxford, 1950, p. 190 sq. 